\title{
THz electromagnetic emission by coherent infrared-active phonons
}

\author{
T. Dekorsy, H. Auer, H. J. Bakker, H. G. Roskos, and H. Kurz \\ Institut für Halbleitertechnik II, Rheinisch-Westfälische Technische Hochschule Aachen, D-52056 Aachen, Germany
}

\begin{abstract}
Coherent phonons are excited in single-crystal tellurium by above-band-gap excitation with femtosecond laser pulses. Coherent infrared-active lattice vibrations give rise to the emission of electromagnetic waves at the phonon frequency. The excitation mechanism is based on the ultrafast buildup of a photo-Dember field and the coupling of a polar lattice mode to this field. The dynamics of the Dember field is obtained from a numerical simulation of the electron-hole plasma dynamics close to the crystals surface. This effect itself gives rise to a broadband emission of $\mathrm{THz}$ radiation from highly absorbing semiconductor surfaces. Details of the generation and the outcoupling of the radiation are discussed.
\end{abstract}

\section{INTRODUCTION}

The generation and detection of coherent phonons in condensed media with femtosecond laser pulses allows a detailed study of the interaction dynamics of optically excited carriers with coherent phonons. Several experiments revealed the possibility to excite coherently optical phonons in different semiconductor materials. Depending on the peculiarities of the interaction of the optical excitation with the lattice, different excitation mechanisms for coherent phonons could be identified. ${ }^{1}$ In polar crystals with large surface fields (GaAs, InP), the generation of coherent LO phonons is based on the ultrafast screening of the surface fields by the photoexcited carriers. $^{2-4}$ In high-temperature superconductors coherent phonons are excited by the breaking of Cooper pairs, ${ }^{1}$ while in other layered materials $(\mathrm{GaSe})$ the excitation process is directly related to an impulsive Raman process. ${ }^{1,5}$ For narrow-band-gap materials like tellurium, it has been shown that a displacive excitation mechanism closely related to the Frank-Condon principle in molecules accounts for the generation of coherent optical phonons. This mechanism is based on the sudden change of the equilibrium position of the lattice atoms after pulsed optical excitation of a dense electron-hole plasma. ${ }^{6,7}$ By this mechanism, only modes of high symmetry can be excited, ${ }^{6,7}$ such as the breathing mode of $A_{1}$ symmetry in Te. We have recently shown that other modes of $E$ symmetry, which are Raman and infrared active, are also coherently excited, but appear in the time-resolved reflectivity changes with an amplitude about two orders of magnitude smaller than the $A_{1}$ mode. $^{8}$ Since the elements of the Raman tensors relevant for the detection of these phonons ${ }^{8}$ differ only by a factor of 2 in amplitude, ${ }^{9}$ the large differences in the detected phonon amplitudes are due to the strong and selective displacive excitation mechanism for the $A_{1}$ mode. In contrast, the $E$ modes are most probably excited via a stimulated Raman process. ${ }^{10}$

THz-emission spectroscopy has opened a way to study transient polarization changes in photoexcited semiconductors. ${ }^{11,12}$ Up to now, THz-emission spectroscopy has been applied for the investigation of coherent electronic phenomena like quantum beats in semiconductors, ${ }^{13-15}$ but not for the detection of coherently excited lattice vibrations. In or- ganic crystals, an oscillatory structure in the $\mathrm{THz}$ emission spectrum has been attributed to a vibrational excitation, ${ }^{16}$ although it is not clear whether intermolecular or molecular vibrations account for the signal. Recently, we have presented the first systematic study of THz-wave emission from coherent polar phonons in a solid. ${ }^{8}$ The material under investigation has been Te. Te is an ideal candidate for an investigation of coherent phonons with time-resolved $\mathrm{THz}$ spectroscopy. Due to the high atomic number and the low symmetry of the crystal, Te exhibits Raman and infrared-active phonons in the frequency range below $4 \mathrm{THz}$, which coincides with the detection bandwidth of gated dipole antennas used for $\mathrm{THz}-\mathrm{emission}$ experiments. In the $\mathrm{THz}$ spectrum emitted from the Te crystal, the strongest high-frequency contribution stems from the coherent excitation of a phonon mode that is only infrared active. ${ }^{8}$ Therefore, Raman processes can be excluded as excitation mechanisms. The displacive excitation mechanism can also be excluded due to the low symmetry of the observed mode. ${ }^{7}$ In this paper we show in detail that the excitation of this mode can be explained by the ultrafast buildup of a photo-Dember field ${ }^{17}$ within the first $100 \mathrm{fs}$ after optical excitation. The Dember field results from different diffusion coefficients of electrons and holes. The buildup of the Dember field itself is a new and strong source of broadband $\mathrm{THz}$ emission from highly absorbing semiconductors. The excitation of coherent phonons via an ultrafast buildup of electric fields is in close analogy to the excitation mechanism based on the ultrafast screening of electric fields as observed in GaAs. ${ }^{2-4}$ Both phenomena are based on displacement currents of the optically excited carriers. We present a detailed numerical study of the generation mechanism of Dember-field-driven phonons and examine the $\mathrm{THz}$ radiation emitted by the Dember field itself.

The paper is organized as follows. In Sec. II we describe the experimental setup for the detection of $\mathrm{THz}$ radiation. In Sec. III we describe the experimental observations such as the polarization and excitation intensity dependence of the emitted radiation. In Sec. IV, we present the theoretical model for the carrier dynamics, the electric-field dynamics, and the emitted $\mathrm{THz}$ radiation and compare the calculation results to the experiment. 


\section{EXPERIMENTAL SETUP AND SAMPLES}

The experimental arrangement is a conventional setup for the detection of $\mathrm{THz}$ emission in reflection geometry. ${ }^{11}$ The sample is excited under $45^{\circ}$ incidence by pulses from a Ti:sapphire laser with $1.7-\mathrm{eV}$ photon energy, a pulse duration of $120 \mathrm{fs}$, and a pulse repetition rate of $78 \mathrm{MHz}$. The coherent $\mathrm{THz}$ radiation is emitted into the direction of the reflected optical beam. The radiation is focused with two paraboloidal mirrors onto a dipole antenna, which is microfabricated on ion-bombarded silicon-on-sapphire (SOS). For efficient collection of the radiation on the antenna, a silicon microlens is mounted on the back side of the sapphire substrate. The dipole length of the antenna is $50 \mu \mathrm{m}$ with a gap in the middle. The gap is illuminated with a time delayed gate pulse. For a given time-delay, the incident $\mathrm{THz}$ electric field biases the dipole antenna and induces a dc current, which is measured with a high-sensitivity current amplifier. Both the amplitude and the direction of the current are obtained. They are a measure of the amplitude and the phase of the incident electric $\mathrm{THz}$ field. To achieve an increased signal-to-noise ratio the pump beam is chopped and a lock-in amplifier is used. The time resolution of this technique is limited by the conductance lifetime in the antenna gap of about $500 \mathrm{fs}$, but the optical pulse duration also leads to a reduction of the detection bandwidth. The best antennas used in the experiments have a maximum responsivity at about $1 \mathrm{THz}$. The highest detectable frequencies are around $4 \mathrm{THz}$. For the calculation of the detected $\mathrm{THz}$ signals we model the antenna characteristics by a current rise time given by the pulse duration and a decay time given by the conductance lifetime in SOS. For an investigation of the polarization of the $\mathrm{THz}$ radiation, a wiregrid polarizer is placed before the antenna. The polarization sensitivity of the antenna itself is complex due to the birefringence of the sapphire substrate.

$\mathrm{Te}$ is a group-VI element and crystallizes in a hexagonal lattice (space group $D_{3}^{4}$ or $D_{3}^{6}$ ) consisting of three atoms per unit cell arranged in a helix along the $c$ axis. Te is a singleelement semiconductor with the highest atomic number of any semiconductor. It exhibits strong infrared-active phonon modes due to the large electronic polarizability of the atoms and the low symmetry of the crystal. ${ }^{18,19}$ The lattice vibrations consist of a fully symmetrical, only Raman-active $A_{1}$ mode $(3.6 \mathrm{THz})$, two degenerate Raman- and IR-active $E$ modes $\left(E_{\mathrm{TO} / \mathrm{LO}}^{\prime}: 2.76 / 3.09 \mathrm{THz}, E_{\mathrm{TO} / \mathrm{LO}}^{\prime \prime}: 4.22 / 4.26 \mathrm{THz}\right)$ and one only IR-active $A_{2}$ mode $\left(A_{2, \mathrm{TO} / \mathrm{LO}}: 2.6 / 2.82 \mathrm{THz}\right){ }^{20}$ The internal polarization of the $E$ modes is oriented perpendicular to the $c$ axis, while the $A$ mode is associated with a polarization parallel to the $c$ axis. The samples are Te single crystals with the surface either parallel, perpendicular, or at $45^{\circ}$ to the $c$ axis. The absorption length at the laser wavelength is $40 \mathrm{~nm}$ for excitation of the surface $\perp c$. The excitation beam with an average intensity of about $100 \mathrm{~mW}$ is focused on a spot of approximately $100 \mu \mathrm{m}$. The maximum electron-hole pair density at the sample surface under these excitation conditions is about $5.5 \times 10^{18} \mathrm{~cm}^{-3}$. For a variation of the excitation density the pump pulse is attenuated.

\section{EXPERIMENTAL RESULTS}

Figure 1 shows the detected electric field under excitation

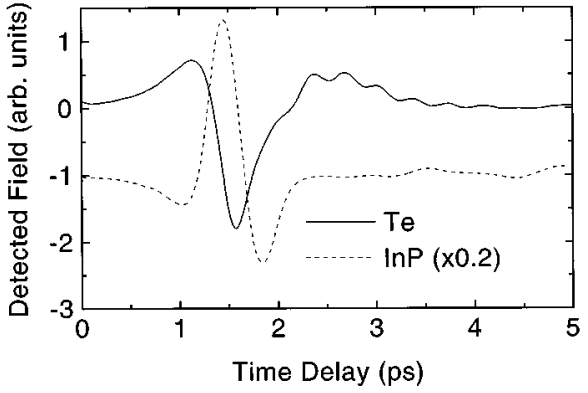

FIG. 1. Detected electric field emitted from the $c \perp$ surface of Te (solid line) and from InP (dashed line). The amplitude of the InP emission is divided by a factor of 5 . The curves are shifted vertically for clarity.

of the $c \perp$ surface (time delay zero is chosen arbitrarily). The emission from a weakly $p$-doped, (100)-oriented InP:Fe sample under the same excitation conditions is shown for comparison. The excitation density in the Te sample at the surface is approximately $5.5 \times 10^{18}$ electron-hole pairs per $\mathrm{cm}^{3}$ at the surface and approximately $6 \times 10^{17} \mathrm{~cm}^{-3}$ in InP. The Te signal consists of a strong initial emission followed by a periodic modulation that is observed for several picoseconds. The strong initial peak is due to a polarization that results from the ultrafast buildup of a strong photo-Dember field. The long oscillating part is due to the coherent emission from an infrared lattice mode. ${ }^{8}$ The oscillating part can be fitted with an exponentially decaying sine function with a frequency of $2.9 \pm 0.05 \mathrm{THz}$ and a dephasing time of 1.6 \pm 0.1 ps. The emission from the InP sample exhibits an initial signal with a duration of 1 ps close to the excitation pulse with a distinctly different shape and a reversed sign compared to the emission from Te. For longer time delays, no emission is observed.

$\mathrm{InP}$ is well known to be a strong broadband emitter of $\mathrm{THz}$ emission. The emission of polar crystals like InP is mainly composed of two effects: (i) an instantaneous signal due to optical rectification in the static surface field ${ }^{21-23}$ and (ii) a signal due to ultrafast displacement currents screening the surface space charge fields. ${ }^{11,24}$ Both effects can be distinguished by their dynamics and polarization orientation. However, at these high carrier densities and the specific excitation geometry both the surface field screening ${ }^{24}$ and the instantaneous polarization take place on the time scale of the pump pulse. The contribution of the buildup of a Dember field in InP is not observed due to the 8 times larger absorption length and the associated lower carrier gradient at the surface. In Te, the screening of depletion-layer fields as the source of the initial radiation can be neglected because the charged surface states have a low density and the band gap is very small $(0.33 \mathrm{eV}) .^{19}$ The surface photovoltage of Te is in the $\mu \mathrm{V}$ regime, ${ }^{25}$ while the surface photovoltage in $\mathrm{InP}$ can be as high as half the band-gap energy of $1.3 \mathrm{eV}$.

The reversed sign in the emitted electric fields is explained as follows. The InP sample is $p$ doped, giving rise to a downward band bending at the surface. The optically excited carriers screen the surface field via drift of the electrons towards the surface and hole drift into the bulk. The Demberfield dynamics in the Te sample is based on a faster diffusion of electrons as compared to holes into the bulk. The Dember field changes in time, until ambipolar diffusion of electrons 


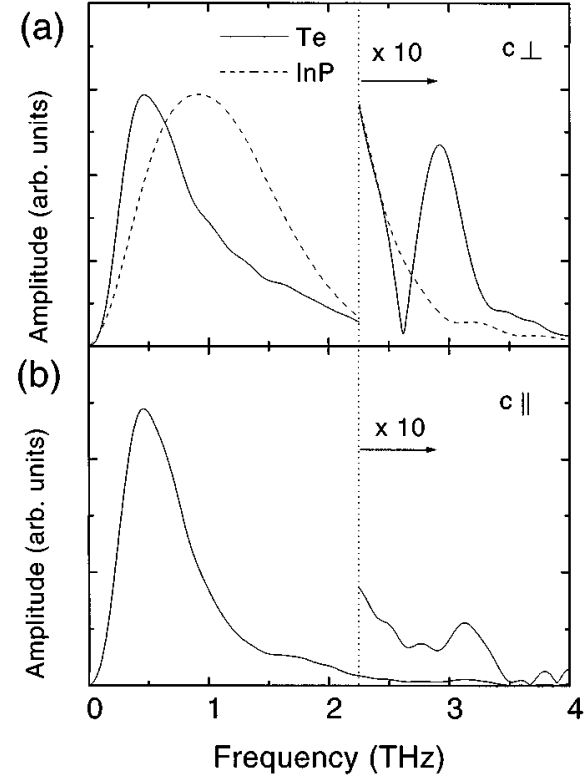

FIG. 2. (a) Amplitude of the Fourier transform of the time domain signal of the Te sample, $c \perp$ surface (solid line) and InP (dashed line) in Fig. 1. The high-frequency part $(>2.25 \mathrm{THz})$ is enlarged by a factor of 10. (b) Fourier transform of the $\mathrm{THz}$ field emitted from the $c \|$ surface. The high-frequency part is also depicted enlarged by a factor of 10 .

and holes is established. The effective dipoles set up by these displacement currents are of different signs. Since the antenna detects the far-field dipole radiation of these polarization changes, the sign is also reversed.

Figure 2(a) depicts the amplitude of the Fourier transforms of the time-domain signals of Fig. 1. Figure 2(b) shows the Fourier transform of the emission from the $\mathrm{c} \|$ surface, which will be discussed below. For the Te sample ( $\perp \perp$ surface), the frequency spectrum reveals a broad peak at approximately $500 \mathrm{GHz}$. For higher frequencies, the signal decreases to nearly zero at $2.62 \mathrm{THz}$ before reaching a second maximum at about $2.9 \mathrm{THz}$. For clarity, this part of the spectrum is enlarged by a factor of 10 . The spectrum of InP has a maximum at $0.95 \mathrm{THz}$ and decreases monotonically with increasing frequencies.

At these high excitation densities, the screening of the surface field in InP is completed during the pulse duration of $120 \mathrm{fs}^{24}$ The maximum of the emission spectrum should be in the range of $8 \mathrm{THz}$, as is verified by calculations presented below. Therefore, the spectrum of the InP sample essentially shows the antenna response. The spectrum of the Te sample deviates from the InP signal significantly in two aspects: (i) The low-frequency maximum is shifted from $0.95 \mathrm{THz}(\mathrm{InP})$ to $0.5 \mathrm{THz}(\mathrm{Te})$ and (ii) the high-frequency part of Te at 2.9 $\mathrm{THz}$ is a factor of 3 larger (value obtained from the nonnormalized spectra) than the amplitude of the broad-band emitter at that frequency. The first observation indicates that the initial strong emission from $\mathrm{Te}$ is not induced by an effect on the time scale of the pump pulse. A transport mechanism has to be introduced with significant slower dynamics. The second observation indicates that the emitted high-frequency radiation at $2.9 \mathrm{THz}$ has an enormously large amplitude. The dip in the Te spectrum at $2.6 \mathrm{THz}$ coincides well with the TO of the $A_{2}$ mode and the peak at $2.9 \mathrm{THz}$ is slightly above the
LO frequency of the same mode. As we will show in Sec. IV C, a slight shift of the emission maximum above the LO phonon frequency is expected from the full theory. Concerning the structure of the spectrum close to the phonon frequencies, the spectrum looks very similar to the recently calculated $\mathrm{THz}$ emission spectrum expected from coherent phonon oscillations in GaAs. ${ }^{4}$

The $A_{2}$ mode has a polarization perpendicular to the excited surface and can be coherently excited by ultrafast field changes with the same orientation. In time-resolved pumpprobe experiments on the same sample, we have verified the coherent excitation of the $A_{1}$ mode and both $E$ modes. ${ }^{8}$ However, the only infrared-active $A_{2}$ mode is not accessible in time-resolved reflectivity measurements in the visible, where the modes appear via their Raman activity. In order to verify the $A_{2}$ mode as the source of the strong highfrequency emission from Te, we investigate the state of polarization of the emitted $\mathrm{THz}$ radiation. For an internal polarization oscillating perpendicular to the excited surface, we expect that the emitted dipole radiation is $p$ polarized with respect to the plane of incidence of the laser pulse. For the measurement of the state of polarization of the $\mathrm{THz}$ radiation we compare the emission without wire-grid polarizer and with the polarizer placed into the $\mathrm{THz}$ beam path. The contrast ratio of the polarizer at $100-\mu \mathrm{m}$ wavelength $(3 \mathrm{THz})$ is better than 100:1. The experimental results are depicted in Fig. 4 of Ref. 8. We observe that the $p$ component of the radiation is very similar to the signal without polarizer. A minor effect of the polarizer is a time delay of $1.5 \mathrm{ps}$ between the signals with and without polarizer. In the $p$-polarized component, both the initial strong emission and the oscillation are observed with almost the same amplitude as in the signal without polarizer. In the $s$-polarized component the initial radiation has changed in shape and is reduced in amplitude by a factor of 4 . No oscillations are observed in the $s$-polarized component for longer time delays. These observations support the assumption that both the strong initial emission and the oscillatory component of the radiation stem from polarization changes oriented perpendicular to the excited surface, i.e., parallel to the $c$ axis. The change in shape and amplitude of the $s$-polarized component strongly indicates that the origin of the emission differs from that of the $p$ component. The Fourier transform of the $s$-polarized component exhibits a maximum at $1 \mathrm{THz}$, which is twice the frequency of the low-frequency maximum in the $p$-polarized and unpolarized spectra and close to the value obtained for InP. Therefore the $s$ component is attributed to the optical rectification effect ${ }^{22}$ based on the bulk $\chi^{(2)}$ of Te.

The Fourier transform of the $\mathrm{THz}$ emission from the sample with the $c \|$ surface is shown in Fig. 2(b). The THz signal is similar to the data shown for the $c \perp$ surface. The only significant difference is that the minimum and the maximum in the high-frequency part of the spectrum both shift by approximately $0.2 \mathrm{THz}$ to higher frequencies. Therefore, the structure can be identified with the $E_{\mathrm{TO}}^{\prime}$ and $E_{\mathrm{LO}}^{\prime}$ mode. The $E^{\prime}$ mode has an internal polarization perpendicular to the excited surface. In the time-resolved pump-probe data, the coherent $E_{\mathrm{TO}}^{\prime}$ and $E_{\mathrm{LO}}^{\prime}$ modes are also observed via their Raman activity. ${ }^{8}$ For the sample with $45^{\circ}$ angle between the surface and the $c$ axis, the spectrum is still dominated by the $A_{2}$ mode. No coupled extraordinary phonons are observed 


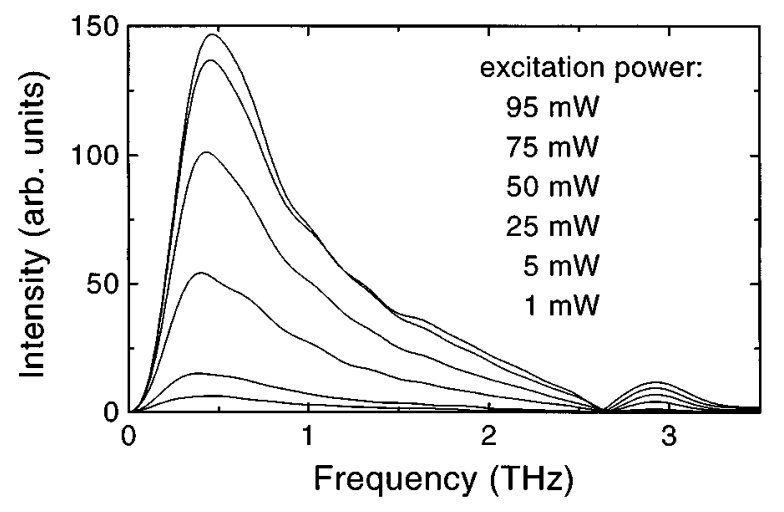

FIG. 3. Fourier transform of the THz field emitted from the $c \perp$ surface for different optical excitation powers as given in the figure.

with frequencies different from the bare LO and TO mode, in contrast with previous Raman scattering experiments. ${ }^{26} \mathrm{We}$ attribute the observation of the $A_{2}$ mode at this surface to the larger oscillator strength of this mode as has been derived from infrared reflectivity data. ${ }^{27}$

For the further investigation of the generation mechanism of the broadband emission and the coherent phonons we perform a variation of the laser intensity. Figure 3 depicts the Fourier transforms of the time domain data under a variation of the excitation intensity over two orders of magnitude from $1 \mathrm{~mW}$ to $95 \mathrm{~mW}$ pump power. The different excitation powers are given in the figure. An increase of the amplitude at the low-frequency and high-frequency maximum with increasing excitation power is clearly observed. In addition, the low-frequency maximum shifts from $400 \mathrm{GHz}$ at the lowest power to $500 \mathrm{GHz}$ at the highest power occurs. The maximum at $2.9 \mathrm{THz}$ does not shift with frequency, but broadens for higher optical powers by a factor of 1.4. The broadening is interpreted as the influence of plasmon-phonon interactions as will be discussed below (Sec. IV D). For the determination of the power dependence of both the initial emission and the emission at the phonon frequency we integrate over the spectra from 0 to $2.6 \mathrm{THz}$ and from 2.6 to 5 THz. The result is shown in Fig. 4. The data are compared to theoretical results that will be discussed in Sec. IV. No significant difference between the excitation density depen-

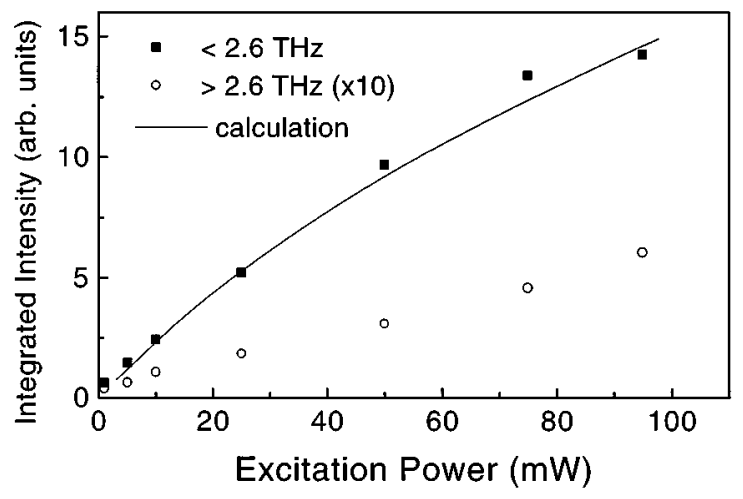

FIG. 4. Value of the integral over the spectra in Fig. 3 from 0 $\mathrm{THz}$ to $2.6 \mathrm{THz}$ (closed squares) and from 2.6 to $5 \mathrm{THz}$ (open circles). The results of numerical calculations are also shown (solid line, discussed in Sec. IV). dence of the Dember field amplitude and phonon amplitude is expected, since the coupling between them is linear in the associated fields (see Sec. IV C). At low densities, both emitted intensities are observed to rise linearly with increased excitation intensity. At higher intensities, the integral of the low-frequency component increases sublinearly, while the high-frequency component still rises linearly. As will be shown later, this slight difference in the low- and high-field dynamics at higher densities is due to the density dependence of the Dember-field dynamics and the influence of the spectral response of the antenna.

\section{THEORETICAL MODEL AND RESULTS}

The theoretical model is based on a one-dimensional driftdiffusion equation for electrons and holes, which is derived from the Boltzmann transport equation in a relaxation time approximation. ${ }^{28}$ Although we are dealing with experiments on a subpicosecond time scale, the relaxation approximation is justified due to carrier-carrier scattering with a typical scattering rate of $10 \mathrm{fs}$ at the densities of our experiments $\left(>10^{18} \mathrm{~cm}^{-3}\right)$. This high scattering rate establishes a thermalized carrier distribution function within $100 \mathrm{fs}$. Therefore the carrier distribution functions can be described by a heated Maxwell-Boltzmann function with an initial excess temperature $T_{i}$ for electrons and holes (index $i$ for electrons and holes) calculated from the photon energy, the band-gap energy, and the band structure. The excess energy of $1.37 \mathrm{eV}$ has to be divided into an excess energy for electrons and holes according to the band structure. The band dispersion in Te is relatively flat, so that the excitation is not well localized in $k$ space. ${ }^{29}$ Therefore, an exact value for the difference in electron and hole temperatures cannot be given. The total optical excess energy corresponds to a temperature of 10600 $\mathrm{K}$, when we relate the excess energy to a carrier temperature of $3 / 2 k T$. For longer times, the excess energy will be transferred to the lattice via carrier-phonon interaction. We guess an initial carrier temperature of 7000 and $3600 \mathrm{~K}$ for electrons and holes, respectively, and a relaxation time for the temperature of 2 ps. This crude estimate for the energy relaxation is based on the values determined in III-V semiconductors. However, there is no knowledge about the exact scattering rates of high-energy carriers in such less common materials as Te. The dependence of the results on these values will be discussed below. The mobilities $\mu_{i}$ are taken from Ref. 20, i.e., $\mu_{e, \| c}=2380 \mathrm{~cm}^{2} / \mathrm{V} \mathrm{s}$ and $\mu_{h, \| c}=1260$ $\mathrm{cm}^{2} / \mathrm{V} \mathrm{s}$ for electrons and holes, respectively, at $293 \mathrm{~K}$ lattice temperature. They are assumed to be independent of the temperature of the distribution function due to the weak band dispersion. These values are about a factor of 2 smaller for transport perpendicular to the $c$ axis. The diffusion coefficients $D_{i}$ are calculated from the generalized Einstein equation, as derived from the fluctuation-dissipation theorem, i.e., $D_{i} / \mu_{i}=k T_{i} / e$. The generation of carriers is calculated according to a Gaussian temporal profile of the laser pulse with a full width at half maximum of $120 \mathrm{fs}$. The spatial profile of the carrier distribution is determined by the absorption length $\alpha^{-1}$ of $40 \mathrm{~nm}$. Bleaching at the pump photon energy during the excitation pulse is neglected as the population is expected to scatter extremely fast out of the initially excited states.

The one-dimensional calculation is justified, since the 


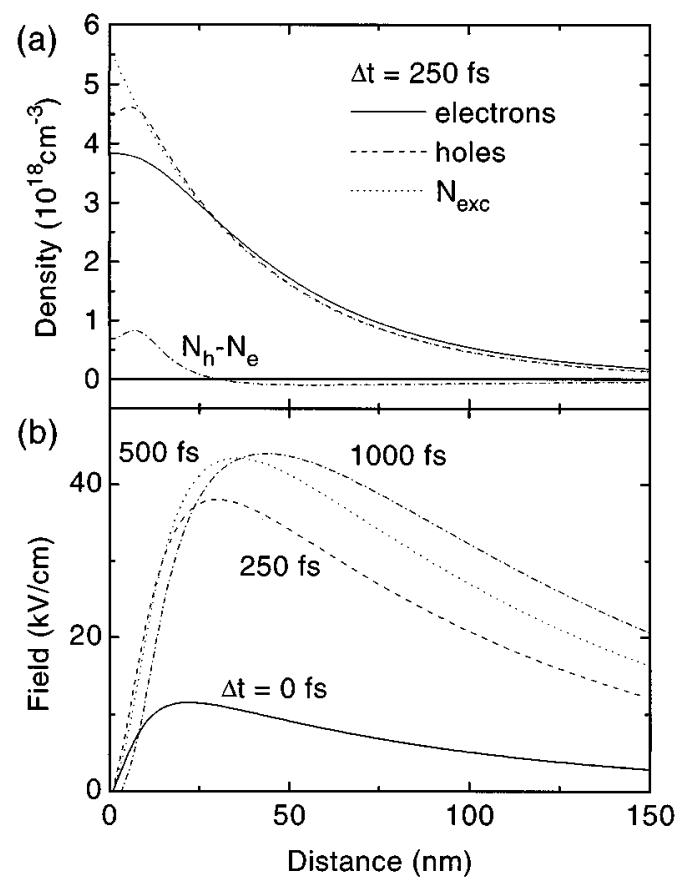

FIG. 5. (a) Calculated spatial distribution of electrons (solid line), holes (dashed line), and the difference of the electron and hole distribution $N_{h}-N_{e}$ (dashed-dotted line) at a time delay of $250 \mathrm{fs}$. The spatial distribution of the excitation density $N_{\text {exc }}$ without considering any carrier transport is shown for comparison (dotted line). (b) Electric-field distribution at different time delays of 0 fs (solid line), $250 \mathrm{fs}$ (dashed), $500 \mathrm{fs}$ (dotted), and $1000 \mathrm{fs}$ (dashed-dotted).

Dember field has only components perpendicular to the excited surface. The carrier gradient parallel to the surface is given by the size of the pump spot of approximately 100 $\mu \mathrm{m}$ as compared to the penetration depth of the optical radiation of $40 \mathrm{~nm}$. Lateral diffusion currents and fields can therefore be excluded on a ps time scale. We note that the same model has been recently successfully applied for the description of electric field dynamics at III-V compound semiconductors surfaces following pulsed optical excitation. $^{24,30}$

\section{A. Dynamics of the electron-hole plasma and the Dember field}

In Fig. 5, results of the numerical simulation are shown. Figure 5 (a) depicts the spatial distribution of electrons and holes at a time delay of $250 \mathrm{fs}$ after the maximum of the excitation pulse. The distribution resulting from the excitation without any drift and diffusion is shown for comparison. After $250 \mathrm{fs}$, the maximum electron density at the surface has already decreased from $5.5 \times 10^{18}-3.8 \times 10^{18} \mathrm{~cm}^{-3}$. The maximum hole density is also reduced by $1 \times 10^{18} \mathrm{~cm}^{-3}$. Both distributions deviate from the generated exponential carrier distribution, as it is calculated from the temporal integral over the laser intensity in the sample neglecting drift and diffusion of carriers. The separation of electrons and holes due to their different diffusivities sets up an effective dipole close to the samples surface. The amplitude of this dipole is given by the difference of the hole and electron distribution $\left(N_{h}-N_{e}\right)$ times their average separation $\Delta z$. The maximum difference in the electron and hole densities of $0.7 \times 10^{18} \mathrm{~cm}^{-3}$ within the first 10 to $20 \mathrm{~nm}$ at the surface is enormously large and comparable to the carrier density difference induced by drift currents in large surface electric fields in polar semiconductors. ${ }^{24}$ The associated space charge field, i.e., the Dember field, set up within the first $250 \mathrm{fs}$ is already large enough to couple the electron and hole diffusion currents. The Dember field establishes ambipolar diffusion for larger time delays. The electric-field distribution at different time delays is shown in Fig. 5(b). Within 500 fs, the electric field reaches a maximum value of $45 \mathrm{kV} / \mathrm{cm}$ at a distance of $25 \mathrm{~nm}$ from the surface. For larger time delays, the maximum of the field does not change significantly. The field maximum propagates into the bulk with a velocity determined by the ambipolar diffusion coefficient of the carriers. A decrease of the Dember field for larger time delays is associated with the cooling of the carriers at a picosecond time scale, when the temperatures of the electron and hole distributions become equal.

A change of the simulation parameters influences the simulation results in the following way. A different choice for the excess energies (temperatures) for electrons and holes influences the maximum field. A higher electron temperature increases the Dember field due to a higher diffusivity of the electrons. Assuming equal initial temperatures for electrons and holes, the maximum Dember field is reduced by a factor of 0.7. The strongest reduction of the Dember field results from a decrease in the initial temperature, a decrease in the absorption length, and a decrease in the carrier density. However, the rise time of the Dember field does not change significantly with these parameters and is several $100 \mathrm{fs}$ also for lower densities. Since the emitted radiation is given by the second time derivative of the polarization, the spectrum of the emitted radiation does not strongly change due to a variation of the parameters, but rather the amplitude is influenced. The choice of the energy relaxation rate also has little effect on the emitted radiation, since it only determines the lowestfrequency components. A variation of the most relevant parameters within the range of uncertainty gives a variation of the maximum Dember field amplitude in the range of a factor of $0.5-1.6$. Even the lowest calculated value of $22 \mathrm{kV} / \mathrm{cm}$ is still comparable to field changes obtained from the screening of surface fields of III-V compounds with doping densities in the range of some $10^{16} \mathrm{~cm}^{-3}$.

\section{B. Emitted and detected THz spectrum}

The radiation detected with the gated dipole antenna is strongly influenced by the complex response function of the antenna. In order to compare the result of the numerical calculations with the experiments we calculate the time dependence of the emitted field and convolute it with the antenna response.

The emitted radiation field $E_{\mathrm{rad}}$ in the far field of the sample is directly related to the second time derivative of the internal field $E_{\text {int }}:{ }^{4}$

$$
E_{\mathrm{rad}}(r, t+r / c)=\frac{\sin \Phi}{\epsilon(\infty) c^{2}} \frac{V}{r} \frac{\partial^{2} E_{\mathrm{int}}}{\partial t^{2}}
$$

where $\Phi$ is the radiation angle, $V$ the radiation volume, and $r$ the distance from the surface to the detector, respectively the distance to the first collimating paraboloidal mirror. Outcoupling of the internal field $E_{\text {int }}$ through the dense carrier 

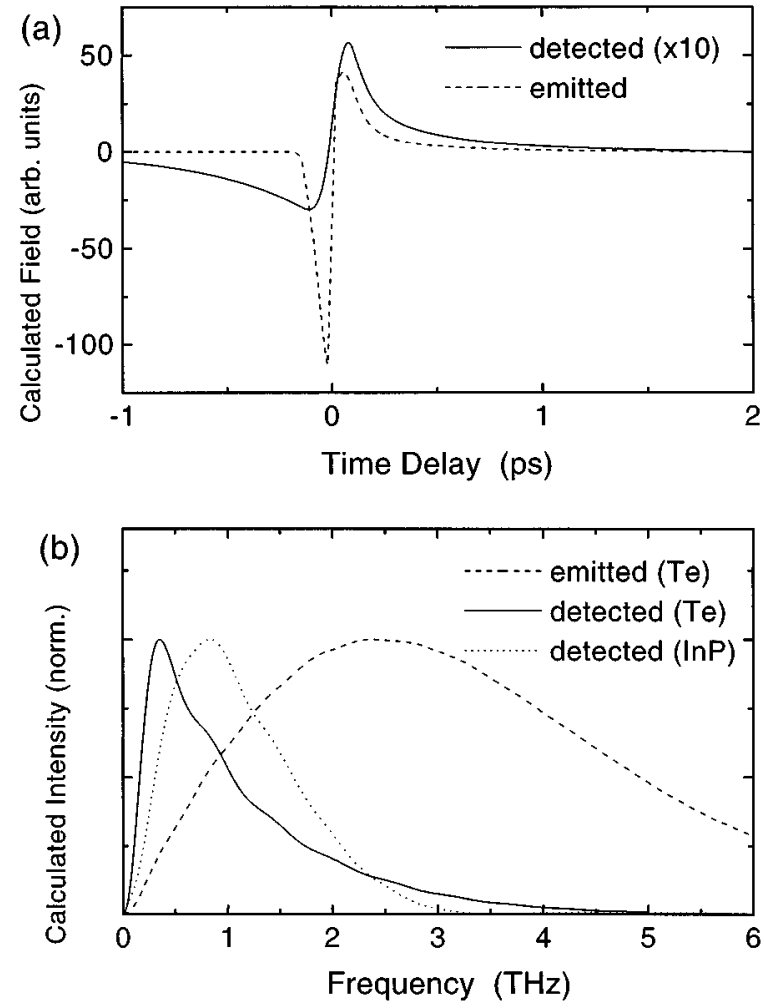

FIG. 6. (a) Calculation of the emitted electric field (dashed line) and the detected signal obtained from convolution with the antenna response (solid line). (b) Fourier transform of the emitted (dashed line) and detected $\mathrm{THz}$ radiation (solid line) of Te. For comparison the detected signal calculated for the emission from an InP surface is also shown (dotted line).

plasma close to the surface and the transmission coefficient through the surface are discussed later. The detected field is calculated by convolution of $E_{\text {rad }}$ with the response function of the antenna. The antenna response is modeled by a rising edge given by the integral over the gate-pulse intensity. The falling edge is given by an exponential decay with a time constant of $500 \mathrm{fs}$ given by the free-carrier lifetime of the optically excited carriers in the SOS antenna gap. Further contributions to the antenna response function such as the impedance and resonances of the antenna are of secondary relevance and are not considered here. ${ }^{31}$

Figure 6(a) shows the calculated emitted and detected fields. Zero time delay is determined by the excitation pulse maximum. The propagation delay $t+r / c$ of the emitted field to the antenna is set to zero in order to allow a direct comparison of the emitted and detected signal. The shape of the initial part of the emitted field is typical for a second time derivative of a field, which develops as the integral over the excitation pulse. However, the asymmetry of the signal indicates a fast initial rise time of the current within the leading edge of the pulse. As soon as the Dember field evolves, the faster diffusion of electrons gets restricted by the drift current in the opposite direction. After $2 \mathrm{ps,} \mathrm{the} \mathrm{quasiequilib-}$ rium of ambipolar diffusion is established, so that no net displacement current is flowing. The THz-emission signal disappears. The convoluted signal strongly changes in shape. The first sharp peak of the emitted signal is strongly decreased in amplitude and is even smaller than the following positive peak. This is a result of the asymmetric antenna response with a slower falling edge. When the gate pulse arrives at negative time delays at the antenna (e.g., $200 \mathrm{fs}$ ) the carrier lifetime of 500 fs leads to an integration over the positive and negative parts of the emitted signal with a slightly stronger contribution from the larger negative part. When the gate pulse arrives at the antenna after the negative part of the emitted field has passed, the free carriers created by the gate pulse are present when only the positiveamplitude field biases the antenna. As a result, the positive field appears with larger amplitude in the detected signal than the leading negative part. Obviously, large initial contributions to $\mathrm{THz}$ radiation signals may be strongly suppressed by the antenna response. This effect has to be considered in the interpretation of $\mathrm{THz}$ signals detected with gated dipole antennas.

Figure 6(b) shows the Fourier transforms of both the emitted and detected signals from the Dember field in Te. The emitted Te signal has a maximum at $2.5 \mathrm{THz}$, which is close to the $A_{2}$ phonon mode. The comparison to the detected spectrum clearly shows that the observed signals are strongly modified by the antenna response. The frequency maximum has shifted to $0.5 \mathrm{THz}$. The cutoff frequency of the signal is at approximately $5 \mathrm{THz}$, which is above the detection limit of $4 \mathrm{THz}$ of the antenna used in the experiments. For comparison, we also calculated the $\mathrm{THz}$ emission from an InP surface with a built-in surface field of $60 \mathrm{kV} / \mathrm{cm}$. The result is also shown in Fig. 6(b). The material parameters of InP are used and the transient field distributions look similar to those calculated for GaAs surfaces. ${ }^{24}$ Optical rectification in the surface field has not been taken into consideration. $^{21,23}$ The amplitude maximum of the emitted electric field due to the ultrafast screening of the surface field is found at $8 \mathrm{THz}$, which is far outside the detection limit of dipole antennas. The maximum amplitude of the detected field is at $0.9 \mathrm{THz}$, which is significantly above the value for $\mathrm{Te}$ and close to the experimentally observed value of 0.95 THz. It is remarkable that the difference of the frequency position of the maxima is conserved when the emission spectra are corrected for the antenna characteristics. In spite of the strong spectral modification by the antenna response, the information on the different time scales of the field dynamics in $\mathrm{Te}$ and $\mathrm{InP}$ is thus retained.

We also investigated the influence of a variation of the excitation power on the emitted signal. The excitation power is varied over the same range as in the experiments. For comparison with the experiments, we calculated the Fourier transform of the detected radiation pulse and integrated over the spectrum. The calculation results are compared to the experimental data in Fig. 4. The calculated values are normalized to fit in amplitude to the experimental values. The calculated density dependence is in good qualitative agreement with the experiment. In addition to the agreement in the emitted amplitude, the calculations also reproduce the experimentally observed shift of the low-frequency maximum from 400 to $500 \mathrm{GHz}$, which is based on a faster rise time of the Dember field at higher excitation densities.

We also modeled the intensity dependence of the radiation spectra for the InP sample (not shown). The frequency shift of the InP emission is stronger compared to the shift calculated and observed for Te. A variation of the excitation flu- 
ence over the same range as for the Te sample results in a calculated shift of the frequency maximum from $0.9 \mathrm{THz}$ to $250 \mathrm{GHz}$, corresponding to a change of the maximum frequency of the emitted radiation from 8 to $1 \mathrm{THz}$. This large shift of the spectrum is expected for the dependence of the dynamics of field screening at different excitation densities. ${ }^{24}$ Screening of a given surface field requires a certain separation of electrons and holes. Assuming saturated drift velocities for the displacement currents, ${ }^{24}$ the field screening develops approximately linearly with time. The higher the excitation density, the sooner the field is completely screened. In contrast, the duration of the buildup of the Dember field is only weakly dependent on excitation density, while the amplitude is strongly influenced. Therefore, the low-frequency emission maximum from the Te sample only weakly changes with the excitation densities, as observed in the experiment.

\section{Coupling of the Dember field to infrared-active phonons}

Up to now we restricted the discussion to the Dember field buildup and the associated emission of $\mathrm{THz}$ radiation. The calculations in the preceding section did show that the Dember field builds up with a maximum frequency component of approximately $2.5 \mathrm{THz}$, which is in the vicinity of the lattice resonances in Te. The free-carrier polarization induced by the Dember effect is oriented perpendicular to the excited surface, so that only infrared-active phonons with polarization oriented into the same direction can directly couple.

In this section, we give a detailed description of the coupling of the Dember field to the phonons and the outcoupling of the $\mathrm{THz}$ radiation at the phonon frequency. The general description is based on the polarization associated with the Dember effect $P_{\text {Dember }}$ and the polarization of the phonon $P$ phonon, which both enter the Maxwell equations as a source term:

$$
\frac{\partial E^{2}}{\partial z^{2}}=-\frac{1}{\epsilon_{0} \epsilon(\omega) c} \frac{\partial P^{2}}{\partial t^{2}}
$$

with the total polarization $P=P_{\text {Dember }}+P_{\text {phonon }}$. Equation (2) is coupled to the equations describing (i) the generation of the polarization of the phonon and (ii) of the Dember polarization due to the different diffusivities of electrons and holes as calculated above. Within the slow-varying amplitude approximation, the polarization of the phonon driven by the electric field is given as ${ }^{32}$

$$
\frac{\partial P_{\text {phonon }}}{\partial t}=-\frac{i N\left|\mu_{12}\right|}{\hbar} E-i\left(\omega-\omega_{\mathrm{TO}}\right) P_{\text {phonon }}-\frac{P_{\text {phonon }}}{T_{2}}
$$

where $\omega_{\mathrm{TO}}$ is the resonance frequency at the TO phonon for a transverse electromagnetic wave, $\mu_{12}$ the transition dipole moment, $N$ the number density of the dipoles, and $T_{2}$ the dephasing time constant. The third relevant equation describes the dynamics of the electronic polarization of the Dember effect, which is described in the preceding section. These three equations form a complete set for the description of the coupled dynamics. In addition, plasmon-phonon coupling can be accounted for by introducing a coupled equation for the lattice displacement and the electronic polarization.
These equations are described in detail in Ref. 4. Furthermore, the outcoupling of the radiation also has to be considered, since it is influenced by the dispersion of the dielectric function in the vicinity of the phonon resonance.

It is important to note that under our experimental conditions the wave vector of the generated $\mathrm{THz}$ light at each frequency in the material parallel to the surface is well defined by the angle of incidence of the incoming light. For an angle of $45^{\circ}$ the transverse wave vector is $444 \mathrm{~cm}^{-1}$ at 3 THz. A small spreading of this transverse wave vector is introduced due to focusing of the incoming beam. This transverse wave vector is very small. The Dember field perpendicular to the surface excited by the optical beam has the same transverse wave vector. As a result of the small transverse wave vector, the phonon polarization and the electric field will dominantly oscillate at the LO frequency, where $\epsilon(\omega)$ has a minimum. The polarization at the TO frequency is small at this wave vector. This consequence is also seen in the basic picture of the phonon-polariton dispersion, where for small wave vectors the electric field is oscillating with the frequency of the upper branch of the dispersion curve close to the LO phonons. ${ }^{33}$ In the experiments at the surface $\perp c$ indeed a slightly shifted frequency of $2.9 \mathrm{THz}$ above the frequency of the LO phonon of $2.82 \mathrm{THz}$ is observed. The nonlinear Dember generation mechanism with a frequency maximum close to the phonon resonance can be regarded as a phase-matched mechanism for the excitation of coherent LO phonons. The solution of the three coupled equations is a computational challenge, since the field and the polarization depend on time and on all three spatial coordinates.

The presence of a dominant polarization at the LO frequency explains the emission of $\mathrm{THz}$ light at this frequency for later times. From this explanation we can exclude the possibility that the observed $\mathrm{THz}$ emission spectrum is explained by absorption of the Dember field emission by the TO phonon. If this would be the case the emission at later times should also oscillate with the TO frequency, which is not observed in the experiments.

Propagation effects of the radiation within the crystal are not important, since the radiating volume is close to the surface [compare Fig. 5(b)] due to the small absorption length at the laser energy. For the absorption length of the $\mathrm{THz}$ radiation at the maximum of the imaginary part of the dielectric constant at the $A_{2}$ mode we calculate $4.8 \mu \mathrm{m}$. The intensity of the $\mathrm{THz}$ radiation generated at a distance of $100 \mathrm{~nm}$ beneath the surface is reduced by only $2 \%$. Therefore the emitting Te volume can be considered as an optically thin sample with respect to the $\mathrm{THz}$ radiation, so that both changes of the amplitude and phase of different frequency components of the radiation by propagation through the crystal can be neglected.

An additional effect is the coupling of the phonons to the plasmons. For a theoretical description of these effect, the two coupled equation for the lattice displacement and the electronic polarizations have to be solved. ${ }^{4}$ Again, the solution of these equations is complicated due to the strong spatial dependence of all involved quantities. The effect of plasmon-phonon coupling on our data is discussed below. Finally, the exact spectrum of the emitted radiation is influenced by the transmission coefficient of the radiation through the Te-air interface. We calculate this effect by using 
Fresnel's law and the real and imaginary parts of the dielectric function. The transmission coefficient is close to 1 for the electric field at the TO phonon frequency and 0.7 at the LO phonon frequency leading to a $30 \%$ reduction of the electric field at the LO frequency outside the material.

\section{THz emission from plasmons}

Recently, the $\mathrm{THz}$ emission from coherently excited phonon-plasmon oscillations in GaAs has been predicted. ${ }^{4}$ The emission of light from longitudinal plasma oscillations is based on the far-field dipole radiation of the oscillating dipole, e.g., as the emission of coherent wave-packet oscillations. ${ }^{15}$ The plasma frequency $\omega_{\mathrm{pl}}$ is given by

$$
\omega_{\mathrm{pl}}^{2}=\frac{N e^{2}}{\epsilon_{0} \epsilon(\infty) m^{*}},
$$

where $N$ is the density, $\epsilon_{0}$ the dielectric permittivity of the vacuum, $\epsilon(\infty)$ the high-frequency dielectric constant, and $m^{*}$ the reduced mass. At a carrier density of $1 \times 10^{18} \mathrm{~cm}^{-3}$ the electron (hole) plasma frequency in $\mathrm{Te}$ is $6.7 \mathrm{THz}(4.8$ $\mathrm{THz}$ ). The values have been calculated using a static effective mass for electrons (holes) of $0.06 m_{0}\left(0.114 m_{0}\right)$ measured at liquid helium temperatures. ${ }^{20}$ However, under the nonequilibrium conditions present in our experiments, the effective mass is lower and high scattering rates also change the frequency. We therefore estimate that the plasma frequency is comparable to the frequency of the coherent phonons observed. Under these conditions, plasmon-phonon coupling is expected to modify the position of the resonances. In addition, the dephasing time of the coupled modes is expected to be faster than that of the unperturbed lattice vibrations due to the momentum relaxation of the charged carriers. For an optically excited electron-hole plasma, both contributions of electrons and holes have to be considered.

In the investigation of the density dependence of the emission, we do not observe a shift of the phonon frequency but only a slight broadening of the peak by a factor of 1.4 when we change the excitation density from approximately $5.5 \times 10^{16}$ to $5.5 \times 10^{18} \mathrm{~cm}^{-3}$. The change in the width of the spectra is associated with a decreasing dephasing time from 2.3 ps at low densities to $1.6 \mathrm{ps}$ at the highest density. Within this density range, the plasma frequency will surely cross the phonon resonance [for the electrons, we calculate the following frequencies: $\omega_{\mathrm{pl}, e} / 2 \pi\left(N=5 \times 10^{16} \mathrm{~cm}^{-3}\right)=1.4 \mathrm{THz}$; $\left.\omega_{\mathrm{pl}, e} / 2 \pi\left(N=5 \times 10^{18} \mathrm{~cm}^{-3}\right)=14 \mathrm{THz}\right]$. However, the carrier density is strongly inhomogeneous due to the short absorption length [see, e.g., Fig. 5(a)]. At the lowest intensities, the optically generated density even varies strongly over a distance that is short compared to the distance that is classically calculated for the displacement associated with the plasma oscillations. An additional spatial inhomogeneity is introduced by the density variation across the laser spot. ${ }^{34,4}$ Due to this inhomogeneities, we do not expect to see a densitydependent shift of the phonon line, but rather a line broadening. When we compare the dephasing time of the $A_{2, \mathrm{LO}}$ mode of 1.6 ps at high excitation densities with the dephasing times of the other coherent modes observed in the timeresolved reflectivity (nonpolar $A_{1}: 2.5 \mathrm{ps;} E_{\mathrm{TO}}$ modes: 10 ps), plasmon-phonon coupling seems to account for the faster dephasing of this mode. For the case of coherent LO phonon excitation in GaAs, no plasmon-phonon coupled modes with frequencies different from the LO or the screened LO phonon at TO frequency have been observed, ${ }^{34}$ which is also an effect arising from the spatial inhomogeneity of the excited carrier distributions. ${ }^{4}$

For the observation of $\mathrm{THz}$ emission by plasmons or plasmon-phonon coupled modes, the excitation and the carrier densities have to be homogeneous. Therefore, plasmonphonon coupling cannot be studied accurately in the $\mathrm{THz}$ emission from Te. Nevertheless, we note the advantage of investigations of coherent plasmon or plasmon-phonon dynamics in time-resolved $\mathrm{THz}$ emission experiments compared to $\mathrm{cw}$ techniques. In Te crystals, plasmon-phonon coupling could only be studied in $p$-doped samples with infrared reflectivity experiments, ${ }^{27}$ since $n$-type doping in Te is not possible. In time-resolved optical experiments a twocomponent plasma is generated, which couples to coherently excited phonons. Dephasing times of these coupled modes can be extracted with subpicosecond time resolution. Recently, coherent plasmon oscillations ${ }^{35}$ and coherent coupled plasmon-phonon oscillations have been observed in bulk GaAs (Refs. 1 and 34) and $\mathrm{GaAs} / \mathrm{Al}_{x} \mathrm{Ga}_{1-x} \mathrm{As}$ heterostructures. $^{36}$ All these excitations should give rise to the strong emission of $\mathrm{THz}$ radiation. However, at the present time the frequency of the optical phonon mode in GaAs is above the detection limit for gated dipole antennas.

\section{E. Radiative lifetime of coherent phonons}

A further dephasing mechanism for coherent infraredactive phonons is the radiative damping of the oscillations. In thermal equilibrium, the lifetime of phonons derived from e.g., cw-Raman experiments is usually determined by the time constant of anharmonic decay. The emission of $\mathrm{THz}$ light by a single LO phonon has very low probability, but for the collective excitation of coherent modes the probability increases strongly. The subject of radiative damping has been discussed recently for the case of coherent charge oscillations in semiconductor heterostructures. ${ }^{37}$ It has been found that the radiative lifetime of the coherent electronic wave packets is in the range of 1000 ps for excitation densities below $10^{-16} \mathrm{~cm}^{-3}$. However, in this system the dephasing of the wave packets due to scattering is in the ps range, which is well below the value of the radiative lifetime. The radiation damping has been found to strongly increase with increasing density due to super-radiance of cooperatively radiating dipoles. ${ }^{37,38}$ When we apply the concept of superradiance to coherently excited phonons, the main difference as compared to the coherent electronic wave packets is the boson character of the phonons. Recently, it has been demonstrated that the generation of coherent phonons allows one to generate a macroscopically large number of phonons in one $\mathbf{q}=0$ mode. $^{39}$ Therefore, a coherently excited infraredactive lattice mode is expected to act as a strong collective emitter of $\mathrm{THz}$ radiation, as observed in our experiments. Our work has also to be regarded in the context of recent proposals for infrared phonon lasers. ${ }^{40}$ Clearly, this subject deserves further experimental and theoretical investigations. 


\section{CONCLUSION}

We demonstrated the excitation of coherent phonons via the ultrafast buildup of photo-Dember fields. This phenomenon is of general interest in the picosecond and subpicosecond dynamics of strongly absorbing media following pulsed optical excitation. For the case of Te, we have shown that infrared-active phonons are driven by the frequency components of the Dember field in the vicinity of the lattice resonances. Both the buildup of the Dember field and the coherent phonons give rise to the emission of electromagnetic radiation in the $\mathrm{THz}$ range, which is detected in the time domain. The dynamics of the Dember field and the associated carrier distributions are calculated on the basis of a driftdiffusion equation including the generation and detection of the $\mathrm{THz}$ radiation. The experimental results on the polarization selection rules and the intensity dependence of the radiation are well explained by the theory. The importance of a detailed modeling of the response function of gated dipole antennas for the interpretation of $\mathrm{THz}$ emission data are demonstrated. The detailed analysis of the coupling of the Dember field to the phonons reveals that the nonlinear Dember field mechanism is a phase-matched excitation mechanism for coherent LO phonons. The effects of plasmonphonon interaction and radiative damping on the dephasing of coherent infrared-active lattice modes have been discussed.

\section{ACKNOWLEDGMENTS}

We gratefully acknowledge A.V. Kuznetsov, G.C. Cho, and S. Hunsche for helpful discussions. We thank $\mathrm{C}$. Waschke for technical assistance. P. Grosse and the 1. Physikalisches Institut of the RWTH Aachen is acknowledged for providing high-quality Te single crystals. This work was supported by the Deutsche Forschungsgemeinschaft.
${ }^{1}$ W.A. Kütt, W. Albrecht, and H. Kurz, IEEE J. Quant. Electron. 28, 2434 (1992).

${ }^{2}$ G.C. Cho, W. Kütt, and H. Kurz, Phys. Rev. Lett. 65, 764 (1990).

${ }^{3}$ T. Pfeifer, T. Dekorsy, W. Kütt, and H. Kurz, Appl. Phys. A 55, 482 (1992).

${ }^{4}$ A.V. Kuznetsov and C.J. Stanton, Phys. Rev. B 51, 7555 (1995).

${ }^{5}$ K. Seibert, H. Heesel, W. Albrecht, J. Geurts, K. Allakhverdiev, and H. Kurz, in The Physics of Semiconductors 3, edited by E.M. Anastassakis and J.D. Joannopoulos (World Scientific, London, 1990).

${ }^{6}$ T.K. Cheng, S.D. Brorson, A.S. Kazeroonian, J.S. Moodera, G. Dresselhaus, M.S. Dresselhaus, and E.P. Ippen, Appl. Phys. Lett. 57, 1004 (1990); T.K. Cheng, J. Vidal, H.J. Zeiger, G. Dresselhaus, M.S. Dresselhaus, and E.P. Ippen, ibid. 59, 1923 (1991).

${ }^{7}$ H.J. Zeiger, J. Vidal, T.K. Cheng, E.P. Ippen, G. Dresselhaus, and M.S. Dresselhaus, Phys. Rev. B 45, 768 (1992).

${ }^{8}$ T. Dekorsy, H. Auer, C. Waschke, H.J. Bakker, H.G. Roskos, H. Kurz, V. Wagner, and P. Grosse, Phys. Rev. Lett. 74, 738 (1995).

${ }^{9}$ A.S. Pine and G. Dresselhaus, Phys. Rev. B 4, 356 (1971).

${ }^{10}$ S. DeSilvetri, J.G. Fujimoto, E.B. Gamble, L.R. Williams, and K.A. Nelson, J. Chem. Phys. 116, 5391 (1985).

${ }^{11}$ X.-C. Zhang and D. H. Auston, J. Appl. Phys. 71, 326 (1992).

${ }^{12}$ H. G. Roskos, in Festkörperprobleme/Advances in Solid State Physics (Vieweg, Braunschweig, 1994), Vol. 34.

${ }^{13}$ H.G. Roskos M.C. Nuss, J. Shah, K. Leo, D.A.B. Miller, A.M. Fox, S. Schmitt-Rink, and K. Köhler, Phys. Rev. Lett. 68, 2216 (1992).

${ }^{14}$ P.C.M. Planken, M.C. Nuss, I. Brener, K.W. Goossen, M.S.C. Luo, S.L. Chuang, and L. Pfeifer, Phys. Rev. Lett. 69, 3800 (1992).

${ }^{15}$ C. Waschke, H.G. Roskos, R. Schwedler, K. Leo, H. Kurz, and K. Köhler, Phys. Rev. Lett. 70, 3319 (1993).

${ }^{16}$ X.-C. Zhang, X.-F. Ma, Y. Jin, T.-M. Lu, E.P. Boden, P.D. Phelps, K.R. Stewart, and C.P. Yakymyshyn, Appl. Phys. Lett. 61, 3080 (1992).

${ }^{17}$ H. Dember, Phys. Z. 32, 554 (1931).

${ }^{18}$ P. Grosse, M. Lutz, and W. Richter, Solid State Commun. 5, 99 (1967)

${ }^{19}$ P. Grosse, The Physics of Tellurium, Springer Tracts in Modern
Physics Vol. 48 (Springer, Berlin, 1969).

${ }^{20} \mathrm{P}$. Grosse and W. Richter, in Physics of Non-tetrahedrally Bonded Elements and Binary Compounds I, edited by O. Madelung, Landolt-Börnstein, New Series, Group III, Vol. 17, Pt. e (Springer, Berlin, 1983).

${ }^{21}$ S.L. Chuang, S. Schmitt-Rink, B.I. Greene, P.N. Saeta, and A.F. Levi, Phys. Rev. Lett. 68, 102 (1992).

${ }^{22}$ X.-C. Zhang, Y. Jin, and X.F. Ma, Appl. Phys. Lett. 61, 2764 (1992); X.-C. Zhang, Y. Jin, L.E. Kingsley, and M. Weiner, ibid. 62, 2477 (1993).

${ }^{23}$ B.B. Hu, E.A. de Souza, W.H. Knox, J.E. Cunningham, M.C. Nuss, A.V. Kuznetsov, and S.L. Chuang, Phys. Rev. Lett. 74, 1689 (1995).

${ }^{24}$ T. Dekorsy, T. Pfeifer, W. Kütt, and H. Kurz, Phys. Rev. B 47, 3842 (1993).

${ }^{25}$ C. Oancea, P. Cristea, E. Zamfir, and M. Popa-Milea, in The Physics of Selenium and Tellurium, edited by E. Gerlach and P. Grosse (Springer, Berlin, 1979), p. 273.

${ }^{26}$ W. Richter, J. Phys. Chem. Solids 33, 2123 (1972).

${ }^{27}$ M. Rautenberg, Ph.D. thesis, Technical University of Aachen, Germany, 1977.

${ }^{28} \mathrm{~W}$. Hänsch, in The Drift-Diffusion-Equation and Its Application in MOSFET Modeling, edited by S. Selberherr (Springer-Verlag, New York, 1991).

${ }^{29}$ K. Maschke and P. Grosse (unpublished).

${ }^{30}$ Y. Rosenwaks, B.R. Thacker, R. K. Ahrenkiel, A.J. Nozik, and I. Yavneh, Phys. Rev. B 50, 1746 (1994).

${ }^{31}$ D. Grischkowsky and N. Katzenellenbogen, Picosecond Electronics and Optoelectronics (Optical Society of America, Washington, 1992).

${ }^{32}$ H.J. Bakker, S. Hunsche, and H. Kurz, Phys. Rev. Lett. 69, 2823 (1992).

${ }^{33}$ M. Born and K. Huang, Dynamical Theory of Crystal Lattices (Oxford University Press, Oxford, 1954).

${ }^{34}$ T. Pfeifer, T. Dekorsy, W. Kütt, and H. Kurz, in Phonon Scattering in Condensed Matter VII, edited by M. Meissner and R.O. Pohl (Springer, Berlin, 1993), p. 110.

${ }^{35}$ W. Sha, A.L. Smirl, and W.F. Tseng, Phys. Rev. Lett. 74, 7555 (1995). 
${ }^{36}$ T. Dekorsy, A.M.T. Kim, G.C. Cho, H. Kurz, A.V. Kuznetsov, and A. Förster, Phys. Rev. B 53, 1531 (1996).

${ }^{37}$ K. Victor, H.G. Roskos, and C. Waschke, J. Opt. Soc. Am. B 11, 2470 (1994).
${ }^{38}$ R.H. Dicke, Phys. Rev. 93, 99 (1953).

${ }^{39}$ A.V. Kuznetsov and C.J. Stanton, Phys. Rev. Lett. 73, 3243 (1994).

${ }^{40}$ R. Brazis, Infrared Phys. Technol. 36, 45 (1995). 